\title{
Determination of the convective heat coefficients by the pulsed photothermal method
}

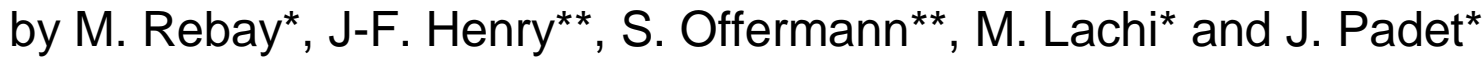 \\ * Université de Reims, Unité de Thermique et Analyse Physique, Laboratoire de Thermomécanique, \\ ** Université de Reims, Unité de Thermique et Analyse Physique, Laboratoire d'Energétique et \\ d'Optique, \\ UFR Sciences, Moulin de la Housse, BP 1039, 51687 Reims, E-mail: mourad.rebay@univ-reims.fr
}

\begin{abstract}
:
We present some elements in order to improve the non-detructive procedure for the measurement of the local heat coefficients between a flat plate and an air flow. We use the pulsed photothermal radiometry method which consists of analysing the transient temperature on the front face of a wall after a sudden deposit of luminous energy by a lump. The infrared camera measures the temperature evolutions at different abscissa from the leading edge of the plate, in order to deduce the local heat coefficients for the same experiment. The results of three identifications, based on a one-dimensional model at any abscissa with constant heat transfer coefficients, are compared.
\end{abstract}

\section{Introduction}

The evaluation of the heat transfer coefficients between a wall and a flow is necessary for the control and the dimensioning of the thermal systems used in the energy processes. The installation of the flux and temperature sensors on the surface of the wall generates, often, considerable disturbances of the heat transfer. This is why the use of a measurement technique without contact has undeniable advantages. For that, we use an pulsed photothermal method. Being able to be employed in-situ, this technique consists in depositing of energy on the front face of the wall, and recording the temporal evolution of the surface. This method was introduced [1 to 3] to determine the thermophysical characteristics of materials, in particular, the thermal diffusivity. It was then extended to evaluate the heat transfer coefficients on the wall subjected to a convective heat exchange [4 to 6] or in the ducts [7]. These authors used a one-dimensional conduction model in the plate and the partial temporal moments of the temperature to identify the heat transfer coefficient.

For the measurement by a transient method the steady state exchange coefficient, it was supposed in above mentioned works that the heat transfer coefficient remains constant during the pulsed experiment. This assumption is debatable if the quantity of energy sent over the wall during the pulse affects considerably the boundary layer. In order to specify the limits of validity of this assumption and the effects of the pulse duration on the results of measurement, we present here a comparative study between the results of three identification methods. All these methods are based on the assumption that the heat transfer coefficient remains constant in time. Moreover, by the use of the infrared camera, we measure the spatial evolution of the convective heat transfer coefficient over the plate.

\section{Description of the experimental set-up}

We realised an experimental set-up (Figure 1) for the measurement, by an infrared camera, of the temporal evolution of the temperature on the front surface of a vertical plate, after a deposit of luminous energy. The experimental device consists of two independent tables. On the first, are assembled the sample and the system of generation of the air flow, and the second comprises the camera and the excitation generator (lumps).

The sample is a $5 \mathrm{~mm}$ thick plastic plate $\left(\mathrm{a}=1,6710^{-7} \mathrm{~m}^{2} \mathrm{~s}^{-1}, \mathrm{~b}=514 \mathrm{~J} \mathrm{~m}^{2} \mathrm{~K}^{-1} \mathrm{~s}^{-1 / 2}\right)$, height of $30 \mathrm{~cm}$ and with $20 \mathrm{~cm}$ width (assumed as a semi-infinite wall). On its front face, we deposited a fine coat of black paint which ensures a maximum emission and absorption of 
the infrared radiation. The flow of an ambient air parallel to the surface of the sample is created by a ventilator. A diffuser of air allows to have a flow covering the totality of the width of the plate. We carried out the acquisition of the transient temperature of the plate for a brief excitation (Dirac) and for three finite duration excitations of the halogens lumps (3.5, 5 and 8 seconds). These measurement are made with three air flows $\left(V_{1}=1.3, V_{2}=2.2\right.$ and $V_{3}=2.9$ $\mathrm{ms}^{-1}$ ), measured on the outlet side of the diffuser of air by a propeller anemometer.

The Dirac excitation is generated by a flash generator. However, the finite duration excitations are generated by two halogens lumps and controlled by an electronic timer. A wood sheet, at the ambient temperature $T_{a m b}$, is placed in front of the lumps after their extinction in order to eliminate the radiation from the lumps (still hot) on the sample during its cooling. The infrared camera is connected to an analogical/digital sampler controlled by the personal computer.

\section{Theoretical model}

The rise in temperature in the semi-infinite wall due to a brief thermal perturbation (Dirac) on its front face, verify, at each abscissa x, the linear model (Figure 2):

$$
\begin{gathered}
\frac{\partial^{2} \theta}{\partial y^{2}}-\frac{1}{a} \frac{\partial \theta}{\partial t}=0 \\
y=0, \quad-\lambda \frac{\partial T}{\partial y}=h \theta(0, t)-W_{f} \delta(t) ; \quad \text { at } t=0, \quad \theta(y, t)=0
\end{gathered}
$$

Where $W_{f}$ is the energy density delivered by the flash lump, $h$ is the global heat exchange coefficient at the abscissa $\mathrm{x}, \delta(\mathrm{t})$ is the Dirac impulsion and $\theta(y, t)=T(y, t)-T_{a m b}$ is the elevation of the temperature at $x$. The use of the one-dimensional conduction model at any abscissa $x$ on the plate is justified by the low thermal conductivity of the plate $\left(\lambda=0.2 \mathrm{~W} \mathrm{~m}^{-1}\right.$ $\mathrm{K}^{-1}$ ) and the uniformity of the energy density distribution. The surface temperature obtained just after the extinction of the flash lump (Figure 3) shows that the energy density is uniformly distributed on the plate.

It was shown in $[4,5]$ that the solution of the system (1) is:

$$
\theta_{d}(0, t)=\frac{W_{f}}{b \sqrt{\pi t}}\left[1-\frac{h}{b} \sqrt{\pi t} \operatorname{exfc}\left(\frac{h}{b} \sqrt{t}\right)\right]
$$

where exfc $(u)$ is a function defined by exp $\left(u^{2}\right)$ erfc $(u)$.

The elevation of temperature for the excitation with a finite duration $t_{c}$ is given by:

$$
\begin{aligned}
& \theta_{c}(0, t)=\frac{\varphi_{f}}{h}\left[1-\operatorname{exfc}\left(\frac{h}{b} \sqrt{t}\right)\right] \quad \text { if } \quad t \leq t_{c}, \\
& \theta_{c}(0, t)=\frac{\varphi_{f}}{h}\left[\operatorname{exfc}\left(\frac{h}{b} \sqrt{t-t_{c}}\right)-\operatorname{exfc}\left(\frac{h}{b} \sqrt{t}\right)\right] \text { if } t \geq t_{c}
\end{aligned}
$$

where $\varphi_{\mathrm{f}}$ is the flux density deposited by the halogens lumps.

\section{Identification methods}

For the identification of the coefficient of transfer we use the partial temporal moments. This notion is developed in [2 to 4]. The analytical expression of the partial zero-order temporal moment of the parietal temperature for a Dirac excitation is: 


$$
m_{0}(t)=\int_{0}^{t} \theta(u) d u=\frac{W_{f}}{h}\left[1-\operatorname{exfc}\left(\frac{h}{b} \sqrt{t}\right)\right]
$$

For a $t_{c}$. excitation, the moment is given by:

$$
\begin{aligned}
& \text { if } \quad t \leq t_{c}, \quad m_{0}(t)=\frac{\varphi_{f}}{h}\left[t-\frac{2 b \sqrt{t}}{h \sqrt{\pi}}-\left(\frac{b}{h}\right)^{2}\left[\operatorname{exfc}\left(\frac{h}{b} \sqrt{t}\right)-1\right]\right] \\
& \text { if } \quad t \geq t_{c}, \quad m_{0}(t)=\frac{\varphi_{f}}{h}\left[t_{c}+\frac{2 b\left(\sqrt{t-t_{c}}-\sqrt{t}\right)}{h \sqrt{\pi}}-\left(\frac{b}{h}\right)^{2}\left[\operatorname{exfc}\left(\frac{h}{b} \sqrt{t}\right)-\operatorname{exfc}\left(\frac{h}{b} \sqrt{t-t_{c}}\right)\right]\right.
\end{aligned}
$$

Direct identification 1. This method consists in seeking, by dichotomy, for an experiment duration $t_{f}$, the theoretical value of $h$ giving the same value of the theoretical zero-order temporal moment, (4) for a Dirac excitation or (5) for a finite duration excitation, as that provided by the experimental thermogram. The knowledge of the energy and flux densities, $W_{f}$ and $\varphi_{f}$ is necessary here.

The energy density $W_{f}$ is given by the flash installation and equals to $12000 \mathrm{~J} \mathrm{~m}^{-2}$. However, the flux density delivered by the halogens lump is evaluated from the thermal response of the plate in free convection. From the cartography of the transient temperature of the plate obtained in natural convection, we choose the temperature at different abscissa $x$ from the leading edge of the plate. For each abscissa, we assimilate the experimental temperature to the theoretical one given by equation (3). Thus, from equation (3), by taking the global heat exchange coefficient varying from $15 \mathrm{Wm}^{-2} \mathrm{~K}^{-1}$ for $\mathrm{x}=0.01 \mathrm{~m}$, to $10 \mathrm{Wm}^{-2} \mathrm{~K}^{-1}$ for $x=0.2 \mathrm{~m}$ [9], we evaluate easily the flux density $\varphi_{\mathrm{f}}$. This estimation gives an average value of $\varphi_{\mathrm{f}}$ equals to $\varphi_{\mathrm{f}}=2000 \pm 80 \mathrm{Wm}^{-2}$.

Direct identification 2. This method has been developed by Crowther [6] for the finite duration excitation only. It differs from the precedent method in the fact that the value of $h$ is obtained from the equality between the theoretical $m_{0}$ and the experimental one of the temperature normalised by its maximum given at the moment $t_{c}$. The theoretical $m_{0}$ of the normalised temperature is:

$$
\begin{aligned}
& t \leq t_{c}, m_{0 n}(t)=\frac{1}{\left[1-\operatorname{exfc}\left(\frac{h}{b} \sqrt{t_{c}}\right)\right]}\left[t-\frac{2 b \sqrt{t}}{h \sqrt{\pi}}-\left(\frac{b}{h}\right)^{2}\left[\operatorname{exfc}\left(\frac{h}{b} \sqrt{t}\right)-1\right]\right] \\
& t \geq t_{c}, m_{0 n}(t)=\frac{1}{\left[1-\operatorname{exfc}\left(\frac{h}{b} \sqrt{t_{c}}\right)\right]}\left[t_{c}+\frac{2 b\left(\sqrt{t-t_{c}}-\sqrt{t}\right)}{h \sqrt{\pi}}-\left(\frac{b}{h}\right)^{2}\left[\operatorname{exfc}\left(\frac{h}{b} \sqrt{t}\right)-\operatorname{exfc}\left(\frac{h}{b} \sqrt{t-t_{c}}\right)\right]\right.
\end{aligned}
$$

The advantage of this method is that the knowledge of the flux density $\varphi_{\mathrm{f}}$ is not necessary, since the expression (6) does not contain the flux density. However, the accurate knowledge of the maximum temperature is important for the calculation of the experimental $\mathrm{m}_{0 \mathrm{n}}$.

Correlation formula. This method is also developed by Crowther [6], witch considered that, at large value of the experiment duration $t_{f}$, the $m_{0}$ of the surface temperature is equal for $a$ Dirac or a finite duration excitation. Consequently, It is interesting to use the Dirac zero-order moment (4) instead of the finite duration excitation one (5) since it is analytically simpler to calculate. The zero-order moment (4) can be written as: 


$$
m_{0}^{*}=\frac{m_{0}(t) b}{W_{f} \sqrt{t}}=\left(\frac{h \sqrt{t}}{b}\right)^{-1}\left[1-\operatorname{exfc}\left(\frac{h}{b} \sqrt{t}\right)\right]
$$

We note the appearance of a relation $\frac{h}{b} \sqrt{t}=f\left(m_{0}^{*}\right)$ such as : $f^{-1}(u)=\frac{1}{u}[1-\operatorname{exf} c(u)]$.

For the values of $m_{0}^{*}$ obtained by the experimental thermograms, we established numerically the correlation formula:

$$
f\left(m_{0}^{*}\right)=9.17-36.28 m_{0}^{*}+62.89\left(m_{0}^{*}\right)^{2}-52.25\left(m_{0}^{*}\right)^{3}+16.6\left(m_{0}^{*}\right)^{4}
$$

Thus, the heat transfer coefficient is deduced from: $h=\frac{b}{\sqrt{t}} f\left(m_{0}^{*}\right)$

\section{Results and interpretations}

Experimental thermograms. We present on figures 4 and 5, the experimental thermograms obtained for various flow rates of the air, at $x=3 \mathrm{~cm}$ from the leading edge of the sample, respectively for a Dirac excitation and a $3.5 \mathrm{~s}$ excitation. We note that there is quite good differentiation of the heat transfer rates when increasing the velocity of the air flow.

Radiation losses. We suppose that the global heat coefficient $\mathrm{h}$ is the sum of an $\mathrm{h}_{\mathrm{c}}$ coefficient (convection losses) and an $h_{r}$ coefficient (radiation losses). Using the linearization of the radiative heat transfer [6], we determine the $h_{r}$ coefficient by:

$$
\left.h_{r}=\varepsilon \sigma\left[T^{2}+T_{a m b}^{2}\right] \mid T+T_{a m b}\right]
$$

$\varepsilon$ is the emissivity of the black paint $(\varepsilon=0.95)$ and $\sigma$ is the Stephan-boltzmann constant.

The application of the eq. (10) shows that the $h_{r}$ coefficient is constant in time and space and have an average value: $h_{r}=6 \mathrm{~W} \mathrm{~m}^{-2} \mathrm{~K}^{-1}$. By each identification method, we calculate the global heat coefficient $\mathrm{h}$, afterwards the convective heat transfer coefficient by: $h_{c}=h-h_{r}$.

Convective heat coefficient $h_{c}$. The heat transfer coefficient are calculated with the experiment duration $t_{f}=60 \mathrm{~s}$, since it had been established in [8] that $h$ tended asymptotically in time to the constant values when $t_{f}$ increased.

Table 1 shows the calculated values of the convective heat coefficient $h_{c}$ at $x=5 \mathrm{~cm}$, with the three identification methods. The relative errors $\Delta h_{c}=\frac{h_{c}-h_{c(\text { Dirac })}}{h_{c(\text { Dirac })}}$ are also presented in this table. We compare the calculated $h_{c}$ values with those obtained by Dirac excitation since the two identification methods (direct identification 1 and correlation formula) give the same value of $h_{c}$, at each $x$ and for each flow velocity. This proves the applicability and the accuracy of these methods for Dirac excitation. A comparison with the results of the literature is impossible here since those were established under conditions different from the ours and for well defined flows. What is not the case here, where we seek to evaluate the heat transfer coefficient between a wall and an air flow with an unknown nature (laminar, turbulent...).

We note from table 1 that the relative error increases with increasing the pulse duration, and more particularly when the air velocity decreases. This observation is available for each abscissa $x$. It should be noted all the same that it is the direct identification 1 which gives the results closest to those obtained for a Dirac excitation $\left(\Delta \mathrm{h}_{\mathrm{c}} \approx-25 \%\right)$, whatever the excitation 
duration and the rate of the air flow. However, the relative error is close to - $45 \%$ for the direct identification 2 owing to the difficulty to evaluate the maximum temperature. The relative error can reach - $40 \%$ for the correlation formula method, because of the assumption, that the $m_{0}$ for the Dirac excitation is the same as for the pulse excitation. Consequently, we estimate that, contrary to the results given in [6], the direct identification 2 and the correlation formula are not adapted when the excitation duration becomes larger. Since the absolute relative errors are superior to $25 \%$ by all the methods, it seems that the constant convective coefficient hypothesis is not valid for the great excitation duration values. On the other hand, the deposit of an energy during a long time disturbs considerably the boundary-layer and creates a time variation of the convective heat coefficient.

The evolution of the convective heat coefficient with the abscissa $x$ is given in (Figure 6). This evolution agrees with the empirical correlation for the turbulent external boundary layer given in [9] ( $h_{c} \approx k x^{-0.2}$, with $\mathrm{k}$ :coefficient depending on the fluid). The turbulence is not due to the great values of the velocity, but to the diffuser geometry used in our experiment.

\section{References}

[1] PARKER W. J., JENKINS R. J., BUTLER C. P. and ABBOTT G. L., "Flash method in determining thermal diffusivity, heat capacity and thermal conductivity", J. Appl. Phys. 32, n 9, 1961, p. 16791684.

[2] DEGIOVANNI A., "Identification de la diffusivité thermique par l'utilisation des moments temporels partiels", High Temperatures-High Pressures 17, 1985, p. 683-689.

[3] BALAGEAS D., KRAPEZ J. C. and CIELO P., "Pulsed photothermal modeling of layered materials", J. Appl. Phys. 59, n 2, 1986, p. 348-357.

[4] BALAGEAS D. et BOSCHER D., "L'évaluation des pertes dans les expériences photothermiques impulsionnelles. Application à la détermination des coefficients de transfert convectif sur des maquettes en soufflerie", CRAS, t.305, Série II, 1987, p. 13-16.

[5] BALAGEAS D., BOSCHER D., DEOM A., FOURNIER J. et HENRY R., "La thermographie infrarouge : un outil quantitatif à la disposition du thermicien", Rev. Gén. Therm., n 322, 1988, p. 501-510.

[6] CROWTHER D. J. and PADET J., "Measurement of the local convection coefficient by pulsed photothermal radiometry", Int. J. Heat Mass Trans. 34, n 12, 1991, p. 3075-3081.

[7] MASSAAD A., DARD J., GERVAIS P. et LAURENT M., "Coefficient d'échange dans une conduite cylindrique. Evaluation expérimentale", Rev. Gén. Therm., n² 286, 1985, p. 731-737.

[8] REBAY M., "Contribution à l'étude de la convection forcée en régime variable - Application à la mesure de coefficients d'échange par méthode impulsionnelle", Thèse de Doctorat de l'Université de Reims, 1998.

[9] KAKAÇ S. and YENER Y., "Convective heat transfer", $2^{\text {nd }}$ edition, CRC Press, Boca Raton, 1955.

Table 1 : Convective heat coefficient at $x=5 \mathrm{~cm}$

\begin{tabular}{|c|c|c|c|c|c|c|c|c|}
\hline & Velocity & Dirac & \multicolumn{2}{c|}{3.5 s excitation } & \multicolumn{2}{c|}{ 5s excitation } & \multicolumn{2}{c|}{ 8s excitation } \\
\hline & & $\mathrm{h}_{\mathrm{c}}$ & $\mathrm{h}_{\mathrm{c}}$ & $\Delta \mathrm{h}_{\mathrm{c}}$ & $\mathrm{h}_{\mathrm{c}}$ & $\Delta \mathrm{h}_{\mathrm{c}}$ & $\mathrm{h}_{\mathrm{c}}$ & $\Delta \mathrm{h}_{\mathrm{c}}$ \\
\hline \multirow{3}{*}{ Identif. 1 } & $\mathrm{V}_{1}$ & 107 & 78 & $-27 \%$ & 80 & $-25 \%$ & 83 & $-22 \%$ \\
\cline { 2 - 9 } & $\mathrm{V}_{2}$ & 148 & 112 & $-24 \%$ & 120 & $-18 \%$ & 111 & $-25 \%$ \\
\cline { 2 - 9 } & $\mathrm{V}_{3}$ & 185 & 143 & $-22 \%$ & 151 & $-18 \%$ & 142 & $-23 \%$ \\
\hline \multirow{3}{*}{ Identif. 2 } & $\mathrm{V}_{1}$ & & 55 & $-48 \%$ & 60 & $-44 \%$ & 71 & $-34 \%$ \\
\cline { 2 - 9 } & $\mathrm{V}_{2}$ & & 84 & $-43 \%$ & 92 & $-38 \%$ & 106 & $-33 \%$ \\
\cline { 2 - 9 } & $\mathrm{V}_{3}$ & & 111 & $-40 \%$ & 120 & $-35 \%$ & 135 & $-28 \%$ \\
\hline \multirow{3}{*}{ Corr. Formula } & $\mathrm{V}_{1}$ & 107 & 77 & $-28 \%$ & 68 & $-36 \%$ & 64 & $-40 \%$ \\
\cline { 2 - 9 } & $\mathrm{V}_{2}$ & 147 & 109 & $-26 \%$ & 96 & $-35 \%$ & 96 & $-35 \%$ \\
\cline { 2 - 9 } & $\mathrm{V}_{3}$ & 186 & 149 & $-20 \%$ & 130 & $-30 \%$ & 125 & $-33 \%$ \\
\hline
\end{tabular}


http://dx.doi.org/10.21611/qirt.2000.018

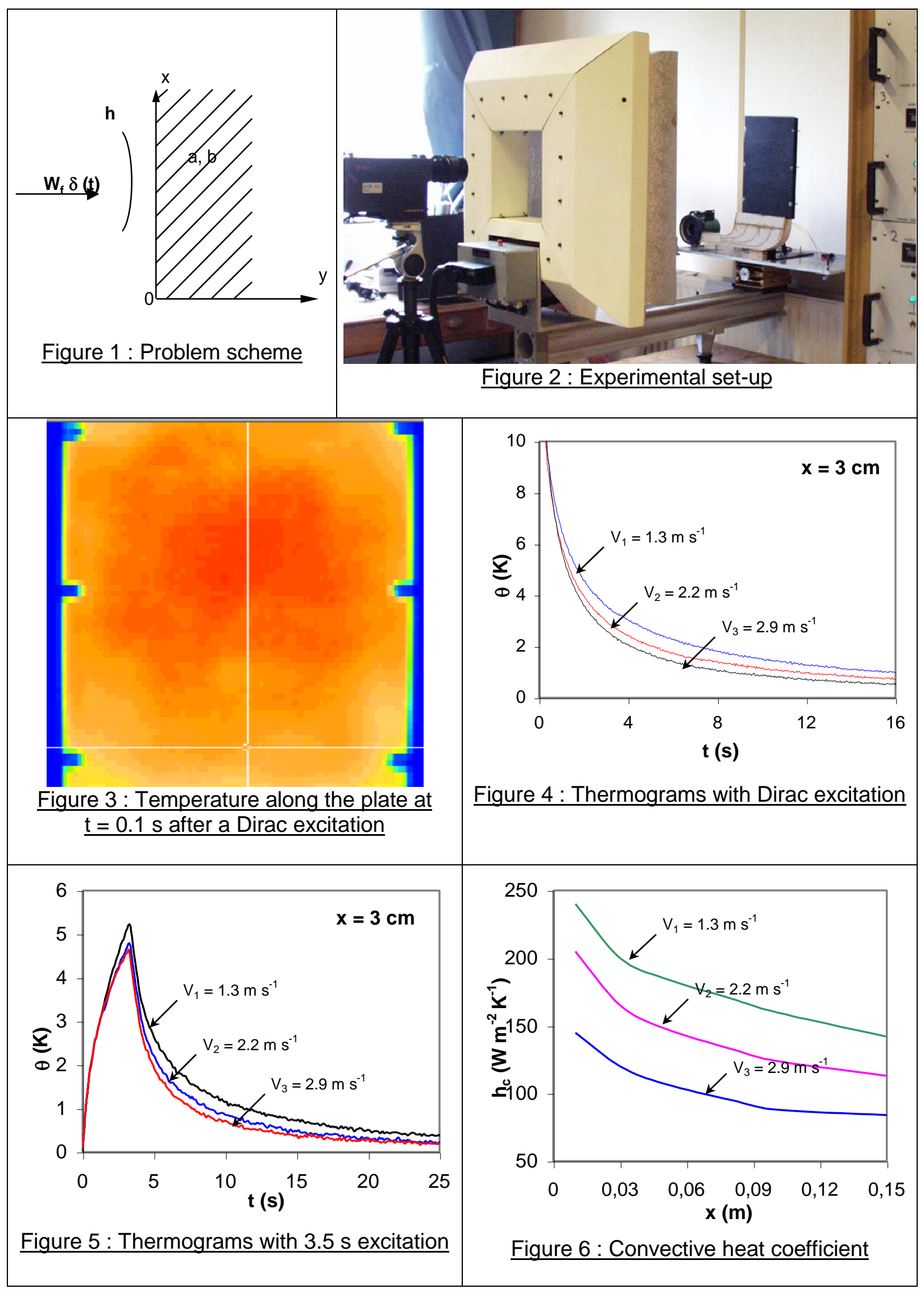

